\title{
The ideal nutritional lifestyle: fasting and rewarding diet
}

\section{Opinion}

What is a diet? It is the continous, stable, established pursuing of a nutritional lifestyle. The world diaita in ancient greek means "way of life". Actually we should live on a diet since what we risk is that all our efforts done in previously followed dietary regimes could vanish when we finally get to our dreamed weight. What we can also say is that a diet must be good in quantity but also in quality: for this reason it is a way of life, a lifestyle, since we should be aware of what we eat. I cited in the article title FASTING since a diet is always based on a certain quantity of fasting, but fasting should not be considered as the total absence of food but it shoud be based on the perception of missing some food we would like to eat, the one permitting us to loose weight or to maintain the weight we need. Fasting has always been present in history: 1 as a religious way of abstinence leading us to better mental states 2 as a way of protest to get rights by some categories of people 3 as a cultural way to stay and be fit.

Infact japanese people think that at each meal you should eat the $80 \%$ of what you think you could eat: for this reason fasting is in this case present too as a partial practice. What $\mathrm{i}$ can say is that fasting, considered like $\mathrm{i}$ told before, has to be applied in most all diets: a loosing weight diet is more or less $2 / 3-3 / 4$ i mean $70 \%$ of our previous nutritional intake. That means that we are doing a $30 \%$ fasting. We feel that from a physical and mental point of view, but a diet doesn't mean feeling weaker, we can also be strong if we do a parallel fitness workout training. Moreover lots of diets are partially hyperproteical: it is tradition to reduce carbohydrates and balance this with more proteins. Insulinemia improves and also the fasting experience is better sustainable. Quality of diet is important: we cannot follow a diet if we don't have guaranteed the correct intake of essential nutrients and of vitamins.

I briefly now compare mediterrenean diet with ketogenic diet, a fasting one: i can summarize by saying that ketogenic diet can be done to loose weight, and then to maintain the body shape hoping also to live longer we should follow the mediterrenean diet. Ketogenic diet is a low carbs relatively hyperproteical diet: chetonic bodies are created due to a somehow acidosis parallel to the told regimen and
Volume 9 Issue I - 2019

\author{
Dora Dragoni \\ Nutritionist, Ordine dei Medici di Bologna, Italy
}

Correspondence: Dora Dragoni, Medical Doctor, Acupuncture fellow, Nutritionist, Personal Trainer, Italy, Tel++39 338508573 I, Email dora.dragoni@studio.unibo.it

Received: January 22, 2019 | Published: January 28, 2019

this is parallel to a partially weaken sensation and in some cases with pollachiuria. But, be sure to loose weight in time. The problems are that not all doctors thinkers agree with that since it can be risky, but lot of sportish people are using it in parallel with fitness good trainings. For these reason its role is controvert but it works. So, fasting is necessary to loose weight: of course chalories intake has be equal and over the basal metabolism need, in order to maintain our organs metabolism and functions and also to be in a guaranteed longevity condition and direction. But it is always suggestable a rewarding diet too: like i said mediterrenean diet can be that target. Be sure to introduce per day some carbs, better if from ancient cereals and whole wheat origins, and also some sweets in a week. It is ideal to maintain a good weight and only if we get rewards we can last on a diet: i can conclude saying that an ideal diet is based on both a small fasting and a reward lifestyle, like for example some chocolate as a snack, or some cake for breakfast. What is important is 1 the total chalories amount 2 the quality of the diet, we should be aware and empowered or follow an expert timetable 3 fasting in a certain percentage meaning this, to sum up: in a day, we should feel hungry also 4 the diet has to reward us in a physical and mental way, as much as we can.

\section{Acknowledgments}

None.

\section{Conflicts of interst}

The author declares that there is no conflicts of interest. 